\title{
Meeting Report: AAPS Workshop on the Role of Dissolution in QbD and Drug Product Life Cycle
}

\author{
Kailas Thakker ${ }^{1,3}$ and Vivian Gray ${ }^{2}$ \\ 'President, Analytical Solutions, Inc., Durham, NC. \\ ${ }^{2}$ Research Editor, Dissolution Technologies, Hockessin, DE.
}

\begin{abstract}
A workshop on the Role of Dissolution in $\mathrm{QbD}$ and Drug Product Life Cycle was held in Crystal City, Virginia. This workshop was jointly sponsored by U.S. Food and Drug Administration and AAPS. The meeting audio tapes are available at http://www. aapspharmaceutica.com/meetings/meeting.asp?id=126.

The theme of the entire workshop was the exploration of the role of dissolution testing in $\mathrm{QbD}$ space. As Tahseen Mirza commented, "Dissolution testing has found its place in drug development in spite of the fact that through QbD, it may be replaced by other tests."

\section{Monday, April 28, $2008 \quad 8$ a.m. to 12:15 p.m. Role of Dissolution in QbD and Product Development Continuum}

\section{Moderators:}

Helen Winkle, U.S. FDA

Tahseen Mirza, Ph.D., Novartis Pharmaceutical

Corporation

Introductory discussions by Helen Winkle and Tahseen Mirza emphasized the role of dissolution testing in a well-designed drug product development program in QbD space. In a well-designed space, dissolution can be replaced by other physical tests. James Polli challenged the need for in vivo testing to assess bioequivalence and showed that in many cases, in vitro studies could be better in terms of total cost and could better assess product performance. Situations where in vitro tests should be viewed as preferable include Class I drugs and Class III drugs with rapid dissolution. Challenges in branded and generic drug product development continue. Since dissolution is a critical quality attribute, it is necessary to understand the formulation, manufacturing, and process parameters and mechanisms, and sources of variability to develop a meaningful test and relevant specification for it. Saji Thomas reported that generics face challenges to comply with $\mathrm{QbD}$ space due to aggressive time lines. On the other hand, there is a wealth of information available for the marketed product.

${ }^{3}$ Corresponding author.

\author{
Monday, April 28, $2008 \quad$ 1:15 p.m. to 5 p.m. \\ Role of Dissolution in QbD
}

\author{
Moderators: \\ Rubin Lozano, Ph.D., Bristol-Myers Squibb Company \\ John Smith, Ph.D., U.S. FDA
}

Arzu Selen spoke on $\mathrm{QbD}$ and its relevance to dissolution-drug release. She emphasized the importance of team efforts in developing novel and predictive dissolution methods for IVIVR or IVIVC. Possible approaches using QbD to develop dissolution tests are: identify desired in vivo performance, estimate desired therapeutic range and maximum variability to develop a dissolution test, continue improving the test during clinical trials. QbD principles facilitate the development of drug products based on knowledge and good science. Paul Dickinson discussed the importance of using the dissolution test as a surrogate for clinical quality. His discussion focused on how clinical quality can be assured using dissolution testing in the QbD space and included several key steps in determining clinical performance. Among these key steps are risk assessment to identify the clinical quality affected and development of a physiologically relevant dissolution test that is most likely to be sensitive to the changes in clinical quality. With proper prior knowledge of the product, dissolution testing can be applied to assure desired clinical quality for a wide range of products in QbD space. Stephen Hammond discussed the use of PAT to map the Dilantin design space and the critical attributes that control dissolution. Critical process attributes that control and can be used to predict dissolution were identified. The combination of DoE and PAT can provide useful information to map design space. John Glennon described two cases where a QbD approach was used for analytical method transfer and validation involving global teams. This resulted in a knowledge repository and platform for future improvements and change control and methods that are better understood and more robust and rugged. Kyle Bui spoke on the use of USP Apparatus 4 and focused beam reflectance measurements for developing a discriminatory dissolution method and a biorelevant dissolution method. 
Tuesday, April 29, $2008 \quad 8$ a.m. to 12 p.m. Relevance of Dissolution in Drug Development

\author{
Moderators: \\ Qingxi Wang, Ph.D., Merck and Company \\ Arzu Selen, Ph.D., U.S. FDA
}

Abu Serajuddin started the day with discussions on the relevance of physicochemical characteristics such as solubility, $\mathrm{pK}_{\mathrm{a}}$, surface area, polymorphism, salt formation, and phase transformation in dissolution and product development. New chemical entities being developed today are at best sparingly soluble in water, making it difficult to develop a traditional dissolution method with aqueous medium. Examples of alternate approaches, such as reducing particle size, choosing an appropriate salt, making use of $\mathrm{pH} / \mathrm{pK}_{\mathrm{a}}$ profile, and selecting an appropriate polymorph, were given. John Crison gave a talk on the role of simulations in dissolution method development. Simulations are necessary to guide product development. Critical parameters such as API particle size, effect of mixing, and other process parameters can be effectively used to aid in simulating in vitro dissolution profile. Jean Surian talked about the role of dissolution in predicting food effects. The presence of food in the Gl tract can have varying type and degree of effect on the absorption of drug. In order to understand the effect of food on drug absorption, dissolution using biorelevant media is a effective tool. Biorelevant media were used to predict the effect of food on several different formulations. Correlations between in vitro dissolution and in vivo performance were obtained. Yun Mao of Merck discussed the importance of biorelevant dissolution in the early phase of drug development. She described five case studies where the use of biorelevant dissolution media aided in formulation selection. Wantanee Phuapradit gave an overview of the role of dissolution method development for generic pharmaceuticals and discussed the differences in parameters that affect in vitro dissolution and in vivo absorption of drugs and the challenges associated with developing IVIVR correlations.

\section{Tuesday, April 29, $2008 \quad$ 1:15 p.m. to 5:30 p.m. IVIVC/R}

\author{
Moderators: \\ Vivian Gray, V.A. Gray Consulting \\ Mansoor Khan, U.S. FDA
}

The afternoon session started with Raimer

Lobenberg's discussion of the use of Gastro Plus ${ }^{\mathrm{TM}}$ to simulate the absorption profiles of drugs in the intestine to compare in vitro dissolution data with clinical data. Two case studies were presented in which Gastro Plus ${ }^{\mathrm{TM}}$ was used to establish IVIVC using flow-thru cells and computer simulations. Examples were also given where the software was used to analyze bioequivalent studies. Charles Tong gave a presentation on the development of a dissolution test for azithromycin sustained-release formulation and the challenges that were overcome in linking the dissolution data with in vivo data to establish IVIVR.

Sandra Klein spoke on the use of biorelevant dissolution media to predict in vivo drug performance. Simplified dissolution media and tests were proposed for highly soluble drug in an immediate-release dosage form. For poorly soluble drugs, simulated intestinal fluids for fasted and fed states were proposed. Examples were given that emphasized the utility of biorelevant dissolution media and how the in vitro data closely correlated with in vivo data. Lawrence Yu of FDA talked about the role of the dissolution test on the extension of biowaivers.

\section{Wednesday, April 30, $2008 \quad 8$ a.m. to 12:30 p.m. Dissolution: Hot Topics}

\section{Moderators:}

Stephen Mayock, Catalent Pharma Solutions William Brown, U.S. Pharmacopeia

William Koch presented the USP perspective on dissolution testing. Since the dissolution test is a performance test that ensures the consistency and equivalency in manufacturing of the clinical trial material, USP is striving to maintain and improve the methodology and performance of existing dissolution equipment. For standardization of dissolution equipment, USP emphasizes the importance of a carefully designed mechanical calibration protocol with periodic performance checks using a performance verification test to ensure that the dissolution test results are consistent and reliable. Joseph Etse presented the dissolution challenges of fixed-dose combination products. Most importantly, the active ingredients have different physical properties. Case studies were presented for hydrochlorothiazide combination products. Jeffrey Lindeman spoke on pharmaceutical co-crystals and the patentability of co-crystals. The definition of co-crystal is still under debate. Co-crystals are the result of synthesis and not recrystallization. Unlike polymers, they are a new composition of matter. Examples of tests to define co-crystals and strategies for patentability were discussed. Vilayat Sayeed discussed the use of the disintegration test as a dissolution test surrogate for highly soluble immediate-release dosage forms and gave examples demonstrating that disintegration is influenced by many process parameters as well as the formulation composition. Eric Duffy talked on the effect of alcohol on the dissolution of extended-release formulations. An example of a potent opiate showed that the presence of alcohol affected the release of the opiate from the dosage form. In vivo data confirmed this effect. The dissolution test was designed to include alcohol in the medium and showed that the presence of alcohol resulted in dose dumping. Mansoor Khan presented a QbD perspective on dose dumping with alcohol. Dose dumping could be 
due to process parameters or some excipients. It is thought that through $\mathrm{QbD}$, one can design a dosage form that would not show dose dumping. For extended-release formulations, if a retardant that is soluble in presence of alcohol is used, dose dumping will occur. Several examples were given where the dissolution of controlled-release formulations was monitored in the presence of alcohol concentrations of $0-40 \%$. Some were vulnerable to the presence of alcohol and others were not. Therefore, failure-mode analysis should be carried out for controlled-release formulations where the presence of alcohol in the system is suspected. 